\title{
A.B.C. approach proposal for POCUS in COVID-19 critically ill patients
}

\author{
Robert Simon $^{1,2}$, Cristina Petrișor ${ }^{1,2}$, Constantin Bodolea ${ }^{2,3}$, Gabriela Csipak ${ }^{1}$, Cristian \\ Oancea $^{1,2}$, Adela Golea ${ }^{1,2}$
}

${ }^{1}$ Clinical Emergency County Hospital, ${ }^{2 “ I u l i u ~ H a t ̦ i e g a n u ” ~ U n i v e r s i t y ~ o f ~ M e d i c i n e ~ a n d ~ P h a r m a c y, ~}{ }^{3}$ Municipal Hospital Cluj-Napoca, Romania

\begin{abstract}
The rapid spread of SARS-CoV-2 (COVID-19) since December 2019 forced Intensive Care Units to face high numbers of patients admitted simultaneously with limited resources. COVID-19 critically ill patients, especially those on mechanical ventilators, demand special attention as they can develop potential complications with critical hemodynamic and respiratory consequences. Point of Care Ultrasound (POCUS) might have important roles in assessing the critically ill SARS-CoV-2 patient. Mostly, lung ultrasound has been presented as having a role in diagnosis and monitoring, but airway examination and hemodynamic evaluation are of interest also.

We propose an A.B.C. POCUS approach focusing on A-airway (orotracheal intubation), B-breathing (interstitial syndromes, pneumothorax, atelectasis, pneumonia), and C-circulation (cardiac function, pulmonary embolism, volume status, deep veins thrombosis). This A.B.C. approach has emerged during ICU care for 22 adult COVID-19 critically ill patients, along with the analysis of recent papers describing ultrasound in COVID-19 patients including the use of ultrasound that is currently applied in the management of the general critically ill population. This A.B.C- POCUS algorithm parallels the wellestablished clinical A.B.C. algorithms. There are few extensive ultrasonographic studies in COVID-19 critically ill patients up to now, but techniques extrapolated from non-COVID studies seem reasonable even though comparative studies are not available yet.
\end{abstract}

Keywords: ultrasound; COVID-19; SARS-CoV-2; critical care; intensive care

\section{Introduction}

The rapid spread of the Novel Coronavirus SARSCoV-2 (COVID-19) from December 2019 led to the declaration of the situation as a Public Health Emergency of International Concern in January 2020 [1]. Healthcare professionals and healthcare systems around the world faced new events, with millions of confirmed cases and hundreds of thousands of deaths worldwide. For the Intensive Care Units (ICUs) around the world, high num-

Received 16.07.2020 Accepted 22.10.2020

Med Ultrason

2021, Vol. 23, No 1, 94-102

Corresponding author: Cristina Petrișor

Department of Anaesthesia and Intensive Care Clinical Emergency County Hospital of Cluj 400006 Clinicilor 3-5, Cluj-Napoca, Romania Tel/Fax: +40 264599438

E-mail: petrisor.cristina@umfcluj.ro bers of patients admitted simultaneously and limited resources that had to be rationalized, put a great deal of pressure on healthcare systems.

COVID-19 critically ill patients, especially those on mechanical ventilators, demand special attention in limited-resource settings regarding available and adequately trained staff, including a time of exposure to all medical personnel. These patients can develop potential complications with critical consequences in terms of hemodynamic and respiratory safety. When caring for COVID-19 patients, the protection of healthcare providers is a real concern and consists of wearing protective equipment that minimises the risk of contamination during medical procedures generating aerosols [2]. However, protective equipment restricts the usual care: lung sounds cannot be heard and stethoscopes are almost impossible to use, e.g. checking for correct endotracheal intubation by listening to lung sounds $[2,3]$. These are important in eve- 
ryday practice in the ICU, but impossible for COVID-19 patients.

Thus, Point of Care Ultrasound (POCUS) could have important roles in assessing the critically ill SARSCoV-2 patient. Ultrasound has proven its utility both in assessing emergency patients (FAST protocol) and monitoring critically ill patients [4]. In the critical care setting, POCUS performed by the Intensive Care specialist has gained significant importance in assuring optimal standards of care. In the current pandemic, the role of POCUS might be even more important as the examination of specific complications is fast, requires minimal training and can be performed in a timely manner in order to re-establish patient physiology by appropriate therapeutic interventions. Mostly, lung ultrasound has been presented as having a role in the diagnosis and monitoring of COVID-19 patients in webinars and training tutorials $[5,6]$. However, there are several other potential applications for the COVID-19 critically ill patient, airway examination and hemodynamic evaluation being included.

Thus, we propose a POCUS approach based on an A.B.C. algorithm (A-airway, B-breathing, C-circulation) that can be useful for the critical SARS-2-COV-19 patient in the ICU.

This A.B.C. POCUS approach has emerged during ICU care for adult COVID-19 critically ill patients admitted to the Anesthesia and Intensive Care I Department of the Clinical Emergency County Hospital Cluj-Napoca in April and May 2020, along with the analysis of recent papers describing techniques and uses of ultrasound in COVID-19 critically ill patients. Also, we included other uses of ultrasound that are currently applied in the management of the critically ill patients overall, but are not specific for COVID-19 patients. A total of 12 papers focusing specifically on ultrasonography in COVID-19 patients were identified and analysed, together with 36 papers selected from the extensive literature focusing on ultrasonography in the general ICU population.

The images were obtained by attending intensivists (RS, CP, GC and CO) with a Philips Lumify S4-1 broadband phased array transducer ${ }^{\circledR}$ (4 to $1 \mathrm{MHz}$ operating frequency range) for abdominal, cardiac and lung applications with preset imaging optimizations (Koninklijke Philips N.V., USA) (Approvals No.14662/2020 and No. 14410/2020).

\section{POCUS-A: Airway}

Endotracheal intubation is a lifesaving procedure if done correctly and represents the fundamental procedure for providing invasive mechanical ventilation. Confirmation of correct endotracheal intubation, and thus ruling out esophageal intubation, is mandatory. This is usually done by the visualization of the endotracheal tube passing through the vocal cords (which might be impossible in the management of the difficult airway), detecting the end-tidal $\mathrm{CO}_{2}$ (which in limited-resource settings might not be available) and clinical auscultation methods. Manual ventilation with a bag might also be detrimental if the patient is not correctly intubated by increasing staff exposure to aerosols. Fiberoptic bronchoscopy may aid in assessing the correct position of the endotracheal tube, but is not feasible in an acute setting given the resources needed and the need for fast confirmation of the correct placement of the endotracheal tube, together with aerosolisation risks. Ultrasound can be a good method for correct endotracheal tube placement [7-9]. Its use can be emphasized for the critical COVID-19 patient. Ultrasound can offer direct and indirect confirmation of correct placement or misplacement of the endotracheal tube.

\section{US examination technique}

Scanning the anterior cervical area above the sternal notch and under the cricoid cartilage with the linear transducer placed in a transverse position offers fast and safe confirmation of the correct placement of the endotracheal tube [7]. The correctly placed endotracheal tube provides a hyperechoic shadow (comet sign). In the case of esophageal intubation, another anatomic structure is present lateral to the trachea (double trachea appearance). An indirect way of assessing the correct placement of an endotracheal tube is scanning for lung sliding during ventilation. Other uses of airway ultrasound are to detect complications of prolonged intubation such as postextubation stridor and subglottic edema, as the cuff leak test increases aerosolization risk and staff exposure, as well as vocal cords paralysis. Though initially presented promising results, the accuracy of ultrasound to predict the presence of subglottic edema and further development of postextubation stridor is a controversial subject $[10,11]$. It has not been evaluated yet for COVID-19 patients.

\section{POCUS-B: Breathing}

Due to potential artifacts that derive from the interaction of sound waves with air filled cavities, for many years the lungs were considered inadequate for ultrasound imagining. However, lung ultrasound has gained a lot of attention in the last two decades, especially in the critical care setting.

Ultrasound is useful for acute pathologies that can suddenly deteriorate, but also to monitor the progression of chronic lung diseases [12-14]. Lung ultrasound findings in different pathologies correlate well with other im- 
aging techniques $[5,6]$. Thus, its use might be an integrated part of POCUS protocols for critically ill COVID-19 patients. Due to the obvious assistance of this technique in critically ill patients, several departments have posted tutorials and webinars on the use of lung ultrasound in COVID-19 patients $[5,6]$.

\section{US examination technique}

When performing lung ultrasound, it is important to distinguish normal versus modified findings. In most patients, with the linear transducer placed over the intercostal space, the pleura is seen as a hyperechoic structure using B-mode, together with the pleural sliding sinchronuous with respiratory movements [12-14]. Horizontal repetitions of the pleural line, the A-lines, represent a reverberation artifact. They are present for the normal lung, but also in pneumothorax. B-lines are hyperechoic comet tail artifacts that start from the pleura, move with respiratory movements and fade into the depth of the image [12-14]. The lung sliding sign, if present, demonstrates that the lung is ventilated. In M-mode, the movement of the lung relatively to the superficial tissue gives the seashore sign (figla) [12-14].

Pneumothorax is a life-threatening condition that needs fast accurate diagnosis and treatment. Obvious causes of acute pneumothorax can lead to a fast diagnosis (e.g. blunt or penetrating trauma to the chest wall), but there are other scenarios where the development of pneumothorax is more subtle, e.g. central venous catheter placement, complication of mechanical ventilation, preexisting lung disease (emphysema bubbles). In COV-

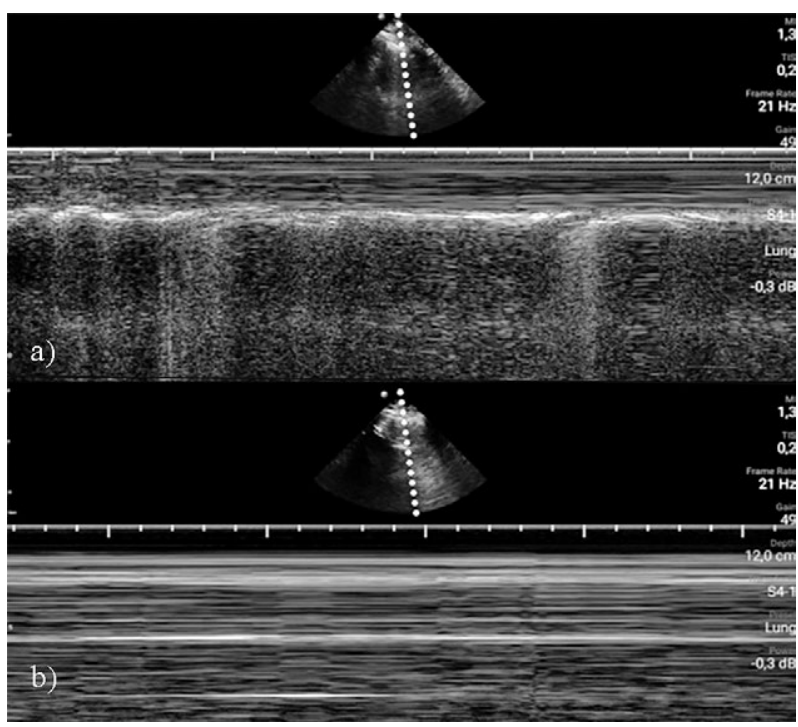

Fig 1. M-mode visualization of the lung in a 60 -years old COVID-19 mechanically ventilated patient with acute right side pneumothorax: left ventilated lung with seashore sign (a), and the stratosphere on the right side of the chest (b).
ID-19 critically ill patients, fast access to bedside radiography (the widely used tool to diagnose pneumothorax) might not be available or might be delayed due to equipment times, and also increases the number of healthcare workers exposed. Also, the use of chest auscultation is not practical due to protective equipment. Because it is accessible, easy to use and accurate, lung ultrasound has proven its utility in diagnosing pneumothorax. Its sensitivity and specificity are higher than those of chest radiography [12-14]. Lung ultrasound is a fast and accurate tool to diagnose this condition [12-14]. There are four ultrasound signs that can help diagnosing acute pneumothorax:

The absence of lung sliding is the first sign. Presence of lung sliding in multiple intercostal spaces means normal lung aeration and rules out pneumothorax. Absence of lung movement evaluated in M-mode gives the stratosphere sign (fig 1b).

Observation of B-lines is important to rule out pneumothorax. If $\mathrm{B}$ lines are present, then pneumothorax is absent.

Pathognomonic for pneumothorax is the lung point sign. The lung point is the point between the partially deflated lung and the inter pleural air cavity formed by the pneumothorax [14]. The lung point can be absent if huge compressive pneumothorax is present. The localization of the lung-point in relation to the scanned areas of the chest wall is correlated well with the extent and severity of the pneumothorax $[12,13]$.

The fourth sign is the absence of a lung pulse. The presence of lung pulse means that the visceral and parietal pleura are in close contact, thus ruling out pneumothorax [14].

Lung ultrasound has comparable results for interstitial syndromes and pneumonia diagnosis versus computed tomography $(\mathrm{CT})$ scans $[12,14]$. The main findings in lung ultrasound in interstitial syndromes are B-lines. Up to three to four B lines in an intercostal space correlate with Kerley-B lines and are called septal rockets. As much as twice the number are called ground glass rockets and correlate with ground glass areas [12]. The presence of B-lines can be focal e.g. pneumonia/pneumonitis, pulmonary contusion, pulmonary infarction, pleural disease or neoplasia, or they can be generalized e.g. pulmonary edema of various causes (cardiac ultrasound recommended to exclude cardiac cause, interstitial pneumonia or pneumonitis, diffuse parenchymal lung disease [13]. B-lines are present in patients with SARS-CoV-2 critical or non-critically ill and correlate well with the progression and resolution of the disease, becoming confluent with progression of disease (fig 2a) [5,6,15-17]. Other findings in SARS-Cov-2 patients are pleural line 


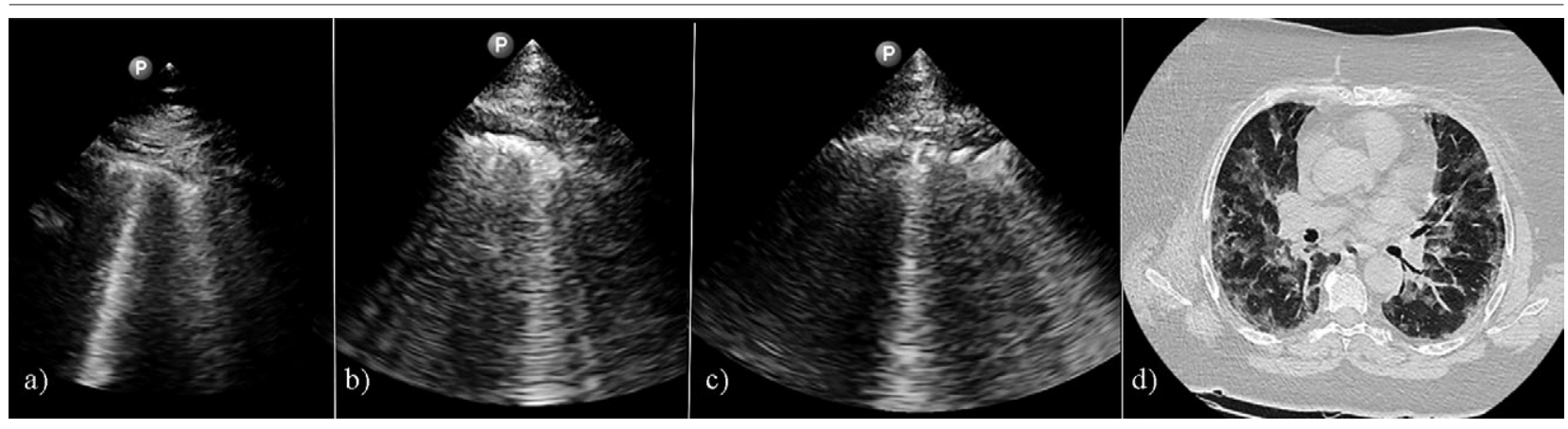

Fig 2. US and computed tomography $(\mathrm{CT})$ images of the lung and pleura in a critically ill COVID-19 patient: the presence of B-lines (a); pleural line thickening (b); sub-pleural consolidations (c); CT image of the lung in the same patient, demonstrating ground-glass opacities (d).

thickening that can be seen as a thick hyperechoic pleural line (fig 2b) and sub-pleural consolidations (fig 2c). Thick irregular pleural lines suggest inflammatory process of the pleura rather than cardiogenic pulmonary edema [6]. Sub-pleural consolidations may leave a comet tail artifact that can be mistaken for a B-line [5,6,15]. Rapid scanning algorithms for suspected COVID-19 patients, might allow the rapid identification of positive patients, especially that imagistic methods seem to have higher diagnostic accuracy compared to Real Time-PCR detection of viral ARN $[18,19]$. US might be easier and accessible, might reveal pathognomonic changes of the lung and pleura, similar to computed tomography (fig $2 \mathrm{~d}$ ).

Although lung consolidations are not pathognomonic in SARS-Cov-2 patients, their presence correlates with disease progression [6]. The sensitivity and specificity of ultrasound for lung consolidations depends on the site, size and depth of the consolidation, with $98 \%$ of consolidations being in contact with the chest wall [20]. The normal aerated lung tissue is replaced by tissue that mimics the aspect of other organs e.g. liver, this being called the tissue-like sign [12]. Other findings can confirm consolidation. The presence of an air bronchogram that is mobile with respiratory fazes can raise the suspicion of pneumonia (fig 3). The air bronchogram appears as punctate hyperechoic structures that are within the consolidation and move in respect to the respiratory fazes [14]. Differentiating lung consolidation and atelectasis can be done using the lung pulse sign which is the transmission of heart beats at the pleural line through a non-inflated lung, this sign being present if the patient has atelectasis [12]. Atelectasis is an important reversible cause for breathing insufficiency and total lung atelectasis with mediastinal shift can be diagnosed using ultrasound or chest radiography (fig 4, Video 1, on the journal site).

Pleural effusions are quite rare in SARS-Cov-2 patients, though frequent in other critical ill patients $[5,6,15]$. About $60 \%$ of critical ill patients have pleural effusions which can prolong mechanical ventilation days and ICU stay [14]. Pleural effusions are easy to spot using ultrasound. The deep boundary of the collection represented by the lung line or the visceral pleura is regular and roughly parallel to the parietal pleura. Ultrasound al-

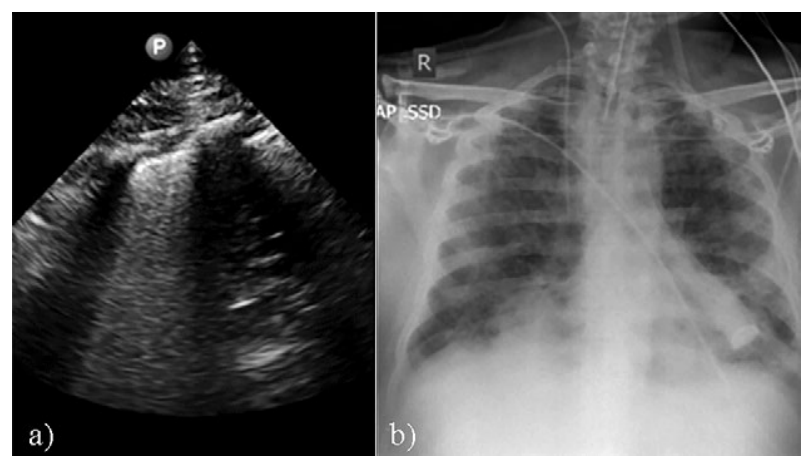

Fig 3. US image of air bronchogram in the left lower lobe in a 66 year-old COVID-19 critically ill patient with bacterial pneumonia (a) compared with chest radiography in the same patient (b).

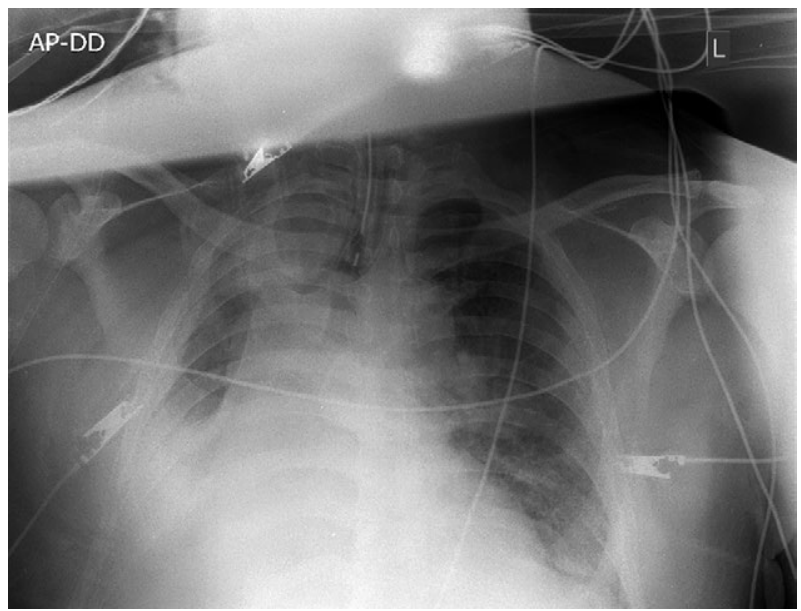

Fig 4. Chest radiography in a 40 year-old critically ill COVID19 patient with right lung atelectasis, displacing the mediastinum to the right. 
lows quantification of the size of the pleural effusion and guides thoracocentesis. Using POCUS like a sono- stethoscope, the intensive care physician can rapidly identify dynamic pleuro-pulmonary changes from SARS-CoV-2 infection.

\section{POCUS-C: Circulation}

Transthoracic POCUS echocardiography has gained ground in the later years as a means for accurate diagnosis and monitoring in the ICU [21-24]. Performed by the intensivist, transthoracic echocardiography has the purpose to identify causes for hemodynamic instability, cardiac arrest included.

\section{US examination technique}

Four views are relatively simple and fast to obtain in a critically ill patient: subcostal, apical four chamber view, parasternal long axis view and parasternal short axis view. Still, special considerations, such as difficulty in patient mobilization and lung inflation during positive pressure ventilation, need attention and might hamper visualisation.

The prevalence of acute myocardial injury, myocarditis included, might be as high as 20\% in COVID-19 patients $[25,26]$. Echocardiography has proven its utility here, demonstrating fast diagnosis in resources' shortages, difficult patient transportation and personal safety reasons. This method can give rapid diagnostic hints when confronting a patient with shortage of breath or sudden hemodynamic deterioration. Fast diagnosis of acute heart failure onset and prompt treatment is possible [27]. Global cardiac function assessment might be depressed in patients with viral infections. A global view of the heart mechanics, thus function, can be obtained from a subcostal approach or an apical four chamber view. Using these windows one can assess the diameters of the heart chambers, as well as wall motion abnormalities $[22,24]$. Left ventricle function, evaluated using the ejection fraction, is important when assessing a patient with shortness of breath, chest pain or sudden drop in arterial blood pressure, aiming to facilitate fast and accurate clinical decision making or therapies.

Right heart failure or dysfunction can occur due to preexisting pathologies such as preexisting chronic obstructive pulmonary disease, obstructive sleep apnea, pulmonary hypertension or acute onset of new disease in the critical ill patient. Also, mechanical ventilation can be an important factor for right heart decompensation. Ultrasound findings are not specific for any of these diseases, but its role becomes important for dynamic monitoring and in acute emergency settings when any delays in treatment leads to poor outcomes [24]. Criti-

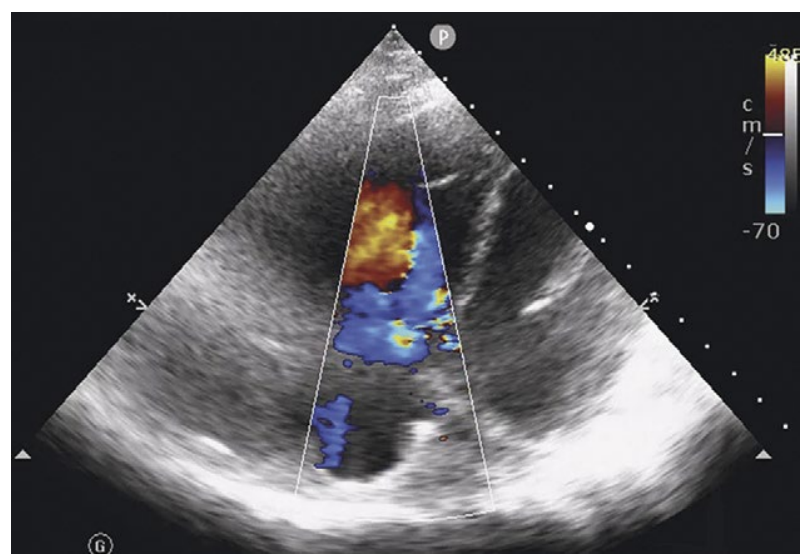

Fig 5. Transthoracic echocardiogram of a 42 year-old patient with massive pulmonary embolism and pulseless electrical activity, demonstrating tricuspid regurgitation, hypokinesis of the right ventricle with acute dilatation.

cally ill patients have a higher risk of developing pulmonary embolism and current data suggests high prevalence for embolic events in SARS-CoV-2 infections [28,29]. Cardiac ultrasound might demonstrate right ventricle enlargement in such patients, the causes being pulmonary embolism, increased pulmonary vascular resistance due to hypoxia or due to aggressive mechanical ventilation [30]. Although transthoracic cardiac ultrasound is not the golden standard to diagnose pulmonary embolism, in the pandemic setting it might be a safe measure of completing the diagnosis, allowing fast treatment [27]. Signs that may be found in a patient with pulmonary thromboembolism are right ventricle hypokinesis with paradoxal septal movement (fig 5), even akinesia of the right ventricle mid free wall with normal motion of the apex. In some cases of massive pulmonary thromboembolism, the left ventricle may be underfilled and hyperdynamic. Right to left ventricle end-diastolic ratio higher than one should guide us towards right ventricle failure [21,24,27,30]. A comprehensive echocardiography performed by a skilled cardiologist may be needed to confirm initial findings.

Patients with SARS-CoV-2 viral infections seem to have prothrombotic status, which might explain why some patients progressed to massive pulmonary thromboembolism. Thromboembolic complications in COVID-19 patients with or without the presence of deep vein thrombosis are frequent and underestimated events due to the lack of active search for deep venous thrombosis [29]. Systematic scanning of peripheral lower extremity veins could diagnose this even in asymptomatic patients. Studies have shown the need for active ultrasound monitoring for deep veins' thrombosis in patients with a history of deep veins thrombosis or pulmonary embolism or who have multiple risk factors [29]. Exclusion is possible 
if veins are compressible, with an adequate blood flow. Visualization of the thrombus in the peripheral venous system, accompanied by the lack of compressibility of the vein on the site of the thrombus confirms diagnosis.

POCUS using multiple views to detect pericardial effusion has high sensitivity and specificity in both medical and trauma patients [24]. Small pericardial effusions can be difficult to detect, but larger ones with hemodynamic impact are easier to diagnose by direct visualization of the hypoechoic pericardial fluid, right atrial or ventricular diastolic collapse, with the significance of increased pericardial pressure. If emergency percutaneous pericardiocentesis is needed, then ultrasound can help guide the needle or choose the best puncture site [24].

Dynamic monitoring of volume status in critical ill patients should be emphasized. It is hard to adequately quantify the circulating blood volume of a healthy patient, but this becomes even more difficult in critical care settings. When evaluating for causes of shock or hypotension, hypovolemia needs to be considered. Inferior vena cava diameter variability in relationship to respiratory phases and central venous pressure is related to intravascular volume status. It provides a good insight into the hemodynamic status in a rapid non-invasive way that can aid clinical decision making [24,31,32]. Especially, fluid overload has negative effects on multiple organs' function with a poor outcome, acute respiratory distress syndromes patients with high vascular permeability, interstitial and tissue edema being at risk for respiratory aggravation. Ultrasound is easy to use for intravascular volume status monitoring and allows therapeutic interventions in real time together with response monitoring to fluid challenges $[33,34]$. Inferior vena cava diameter is measured with the transducer placed in sagital position and inspiratory and expiratory variation in response to respiration is measured, obtaining the distensibility or the collapsibility index, depending on the type of breathing: spontaneous or mechanical [24,34].

\section{Diagnostic accuracy of POCUS in detecting respiratory and hemodynamic disturbances associated with COVID-19 critically ill status}

Being a novel disease, extensive ultrasonographic studies in critically ill patients are lacking. The performance of POCUS in estimating the diagnostic accuracy of the above scanning techniques or algorithms can only be extrapolated from non-COVID patients and studies. Each of these have already been evaluated and meta-analyses on the diagnostic accuracy of each ultrasonographic parameter are currently available. Their application in critically ill COVID-19 patients might seem reasonable, even though comparative studies are not available yet. In the case of unstable patients, we need to reassess $A B C$ and bedside POCUS examination is becoming a new tool that answers clinical questions and guides towards fast decisions.

A. For the confirmation of endotracheal tube placement during airway management, the pooled sensitivity and specificity of POCUS was estimated as being 0.93 [0.86-0.96] and the specificity was 0.97 [0.95-0.98] [35]. Later on, a subsequent meta-analysis comprising 30 studies with more than 2500 patients estimated these values to be around 0.98 [0.971-0.988] and 0.957 [0.901-0.98], respectively, highlighting that ultrasonography is a valuable and reliable adjunct for endotracheal tube confirmation [36].

B. Pneumothorax can be suspected clinically and by ultrasound evaluation of ultrasonographic artefacts. The diagnostic accuracy of lung ultrasound to detect or confirm pneumothorax, as investigated in studies subsequently evaluated in several meta-analysises, is optimal and reaches sensitivity higher than $80 \%$ and specificity higher than $90 \%$ [37-42]. Thus, the diagnosis of pneumothorax using ultrasound is accurate and reliable. Similar values were found for the diagnosis of lung consolidations, pneumonia included $[43,44]$. Even higher values, with over $90 \%$ sensitivity and almost $98 \%$ specificity were found for pleural effusions and interstitial syndromes $[43,45,46]$.

C. The diagnosis of pulmonary embolism mainly relies on CT scanning in current algorithms. Still, ultrasound is a diagnostic alternative for special clinical settings such as patients with respiratory and hemodynamic instability that cannot be transported to the CT units or during cardiac arrest. The sensitivity of right heart strain is quoted to be approximately $53 \%$ sensitive and $83 \%$ specific [47]. For the detection of deep vein thrombosis, proximal veins compression has a sensitivity of $90 \%$ and specificity of $98.5 \%$ [48], but the sensitivity to detect pulmonary embolism is low [49]. However, identification of patients with deep vein thrombosis allows therapeutic anticoagulation commencement. Inferior vena cava diameter and respiratory variability are currently used in the evaluation of volume status in critically ill patients, in integrated decision-making processes for patient management. Fluid responsiveness is predicted with a pooled sensitivity of around $60-80 \%$ and specificity of approximately 70-90\% [50-54], other clinical and hemodynamic parameters being required and simultaneously assessed in this population.

Whether these diagnostic accuracy assessments in the general ICU population can be applied for the critically ill COVID-19 patients, remains to be investigated. 


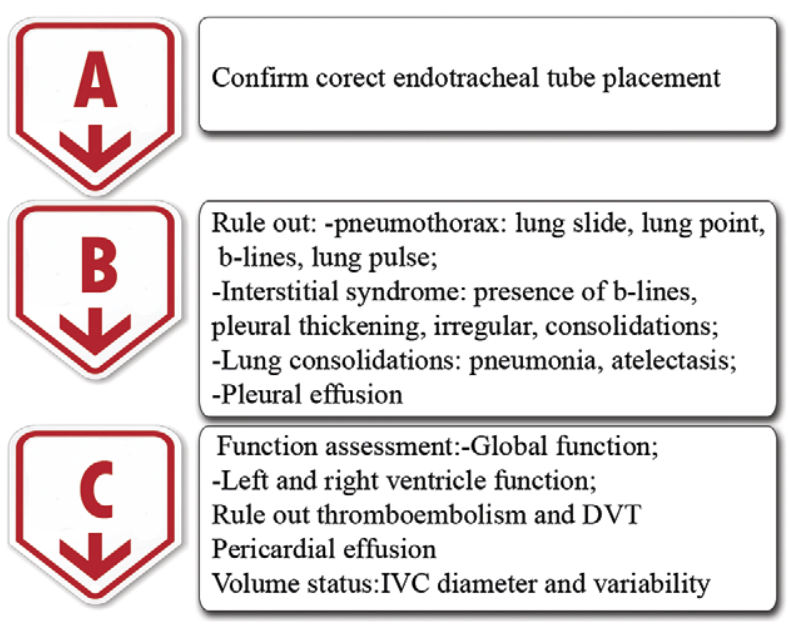

Fig 6. POCUS algorithm for COVID-19 critically ill patients.

\section{Conclusions}

For critically ill COVID-19 patients and healthcare systems confronting pandemics, management of resources, limiting healthcare professionals and healthcare spaces contamination risk (like CT scanners) are of the utmost importance. Also, similar to other critically ill patients, the risk associated with transporting out of the ICU, needs to be minimised. Using a systematic POCUS A.B.C. approach in COVID-19 critically ill patients, similar to the one we propose (fig 6), might reduce the risk of contamination of healthcare workers, thus reducing the exposure risk and the use of hospital resources. POCUS adds the advantages of being non-invasive and non-irradiating, providing real time assessment and relative ease of access. It ensures a fast, safe and resource means of diagnosis.

This POCUS - A.B.C. algorithm parallels the wellestablished clinical A.B.C. algorithms. There are few extensive ultrasonographic studies in COVID-19 critically ill patients up to now, but techniques extrapolated from non-COVID studies seem reasonable even though comparative studies are not available yet.

Acknowledgements: We thank the Beard Brothers for the support with the Philips LumifyS4-1 broadband phased array transducer and all colleagues attending critically ill COVID-19 patients in the department.

\section{Conflict of interest: none}

\section{References}

1. Eurosurveillance editorial team. Note from the editors: World Health Organization declares novel coronavirus (2019-nCoV) sixth public health emergency of interna- tional concern. European Centre for Disease Prevention and Control (ECDC), Stockholm, Sweden, 2020. Available at: https://www.eurosurveillance.org/content/10.2807/15607917.ES.2020.25.5.200131e

2. Cheung JC, Ho LT, Cheng JV, Cham EYK, Lam KN. Staff Safety During Emergency Airway Management for COVID-19 in Hong Kong. Lancet Respir Med 2020;8:e19.

3. Buonsenso D, Pata D, Chiaretti A. COVID-19 outbreak: less stethoscope, more ultrasound. Lancet Respir Med 2020;8:e27.

4. Zieleskiewicz L, Muller L, Lakhal K, et al. Point-of-care ultrasound in intensive care units: assessment of 1073 procedures in a multicentric, prespective, observational study. Intensive Care Med 2015;41:1638-1647.

5. Peng QY, Wang XT, Zhang LN; Chinese Critical Care Ultrasound Study Group (CCUSG). Findings of lung ultrasonography of novole corona virus pneumonia during the 2019-2020 epidemic. Intensive Care Med 2020;46:849-850.

6. Yasukawa K, Minami T. Point-of-care Lung Ultrasound Findings in Patients with COVID-19 Pneumonia. Am J Trop Med Hyg 2020;102:1198-1202.

7. Muslu B, Sert H, Kaya A, et al. Use of sonography for rapid identification of esophageal and tracheal intubations in adult patients. J Ultrasound Med 2011;30:671-676.

8. Men XQ, Yan XX. Tracheal Ultrasound for the Accurate Confirmation of the Endotracheal Tube Position in Obese Patients. J Ultrasound Med 2020;39:509-513.

9. Drescher MJ, Conard FU, Schamban NE. Identification and description of esophageal intubation using ultrasound. Acad Emerg Med 2000;7:722-725.

10. Ding LW, Wang HC, Wu HD, Chang CJ, Yang PC. Laryngeal ultrasound: a useful method in predicting post-extubation stridor. A pilot study. Eur Respir J 2006;27:384-389.

11. Patel AB, Ani C, Feeney C. Cuff leak test and laryngeal survey for predicting post-extubation stridor. Indian J Anaesth 2015;59:96-102.

12. Lichtenstein D. Lung Ultrasound in the critically ill. Curr Opin Crit Care 2014;20:315-322.

13. Volpicelli, G, Elbarbary M, Blaivas M, et al. International evidence-based recommendations for point-of-care lung ultrasound. Intensive Care Med 2012;38:577-591.

14. Mayo PH, Copetti R, Feller-Kopman D. et al. Thoracic ultrasonography: a narrative review. Intensive Care Med 2019;45:1200-1211.

15. Smith MJ, Hayward SA, Innes SM, Miller A. Point-of-care lung ultrasound in patients with COVID-19 - a narrative review. Anaesthesia 2020;75:1096-1104.

16. Volpicelli G, Gargani L. Sonographic signs and patterns of COVID-19 pneumonia. Ultrasound J 2020;12:22.

17. Volpicelli G, Lamorte A, Villen T. What's new in lung ultrasound during the COVID-19 pandemic. Intensive Care Med 2020;46:1445-1448.

18. Long C, Xu H, Shen Q, et al. Diagnosis for the Coronavirus Disease (COVID-19): rRT-PCR or CT? Eur J Radiol 2020;126:108961.

19. He JL, Luo L, Luo ZD, et al. Diagnostic performance between CT and initial real-time RT-PCR for clinically sus- 
pected 2019 coronavirus disease (COVID-19) patients outside Wuhan, China. Respir Med 2020;168:105980.

20. Lichtenstein D, Lascols N, Mezičre G, Gepner A. Ultrasound Diagnosis of Alveolar Consolidation in the Critically Ill. Intensive Care Med 2004;30:276-281.

21. Nagre AS. Focused-Assessed Transthoracic Echocardiography: Implications in Perioperative and Intensive Care. Ann Card Anesth 2019;22:302-308.

22. Faris JG, Veltan MG, Royse CF. Limited transthoracic echocardiography assessment in anaesthesia and critical care. Best Pract Res Clin Anaesthesiol 2009;23:285298.

23. Jensen MB, Sloth E, Larsen KM, Schmidt MB. Transthoracic echocardiography for cardiopulmonary monitoring in intensive care. Eur J Anaesthesiol 2004;21:700-707.

24. Labovitz AJ, Noble VE, Bierig M, et al. Focused cardiac ultrasound in the emergent setting: a consensus statement of the American Society of Echocardiography and American College of Emergency Physicians. J Am Soc Echocardiogr 2010;23:1225-1230.

25. Bavishi C, Bonow RO, Trivedi V, Abbott JD, Messerli FH, Bhatt DL. Acute myocardial injury in patients hospitalized with COVID-19 infection: A review, Prog Cardiovasc Dis 20206 June. doi:10.1016/j.pcad.2020.05.013.

26. Kim IC, Kim JY, Kim HA, Han S. COVID-19-related myocarditis in a 21-year-old female patient. Eur Heart J 2020;41:1859.

27. Zhang L, Wang B, Zhou J, Kirkpatrick J, Xie M, Johri AM. Bedside Focused Cardiac Ultrasound in COVID-19 from the Wuhan Epicenter: The Role of Cardiac Point-of-Care Ultrasound, Limited Transthoracic Echocardiography, and Critical Care Echocardiography. J Am Soc Echocardiogr 2020;33:676-682.

28. Wichmann D, Sperhake JP, Lütgehetmann M, et al. Autopsy Findings and Venous Thromboembolism in Patients With COVID-19: A Prospective Cohort Study. Ann Intern Med 2020;173:268-277.

29. Danzi GB, Loffi M, Galeazzi G, Gherbesi E. Acute pulmonary embolism and COVID-19 pneumonia: a random association? Eur Heart J 2020;41:1858.

30. Szekely Y, Lichter Y, Taieb P, et al. The Spectrum of Cardiac Manifestations in COVID-19: a Systematic Echocardiographic Study. Circulation 2020;142:342-353.

31. Lodigiani C, Iapichino G, Carenzo L, et al. Venous and arterial thromboembolic complications in COVID-19 patients admitted to an academic hospital in Milan, Italy. Thromb Res 2020;191:9-14.

32. Dagadaki O, Birbas K, Mariolis T, Baltopoulos G, Myrianthefs P. Necessity of the Periodical Ultrasound Assessment of the Peripheral Venous System in intensive Care Unit Patients. Ultrasound Med Biol 2019;45:367-373.

33. Ciozda W, Kedan I, Kehl DW, Zimmer R, Khandwalla R, Kimchi A. The efficacy of sonographic measurement of inferior vena cava diameter as an estimate of central venous pressure. Cardiovasc Ultrasound 2015;14:33.

34. KoratalaA, Ronco C, KazoryA. Need for Objective Assessment of Volume Status in Critically Ill Patients with COV-
ID-19: The Tri-POCUS Approach. Cardiorenal Medicine 2020;10:209-216.

35. Chou EH, Dickman E, Tsou PY, et al. Ultrasonography for confirmation of endotracheal tube placement: a systematic review and meta-analysis. Resuscitation 2015;90:97-103.

36. Sahu AK, Bhoi S, Aggarwal P, et al. Endotracheal Tube Placement Confirmation by Ultrasonography: A Systematic Review and Meta-Analysis of More than 2500 Patients. J Emerg Med 2020;59:254-264.

37. Dahmarde H, Parooie F, Salarzaei M. Accuracy of Ultrasound in Diagnosis of Pneumothorax: A Comparison between Neonates and Adults-A Systematic Review and Meta-Analysis. Can Respir J 2019;2019:5271982.

38. Staub LJ, Biscaro RRM, Kaszubowski E, Maurici R. Chest Ultrasonography for the Emergency Diagnosis of Traumatic Pneumothorax and Haemothorax: a Systematic Review and Meta-analysis. Injury 2018;49:457-466.

39. Ebrahimi A, Yousefifard M, Mohammad Kazemi H, et al. Diagnostic Accuracy of Chest Ultrasonography versus Chest Radiography for Identification of Pneumothorax: a Systematic Review and Meta-analysis. Tanaffos 2014;13:29-40.

40. Alrajab S, Youssef AM, Akkus NI, Caldito G. Pleural Ultrasonography versus Chest Radiography for the Diagnosis of Pneumothorax: Review of the Literature and Meta-analysis. Crit Care 2013;17:R208.

41. Alrajhi K, Woo MY, Vaillancourt C. Test Characteristics of Ultrasonography for the Detection of Pneumothorax: a Systematic Review and Meta-analysis. Chest2012;141:703-708.

42. Ding W, Shen Y, Yang J, He X, Zhang M. Diagnosis of pneumothorax by radiography and ultrasonography: a meta-analysis. Chest 2011;140:859-866.

43. Staub LJ, Mazzali Biscaro RR, Kaszubowski E, Maurici R. Lung Ultrasound for the Emergency Diagnosis of Pneumonia, Acute Heart Failure and Exacerbation of COPD/ asthma in Adults: A Systematic Review and Meta-analysis. J Emerg Med 2019;56:53-69.

44. Alzahrani SA, Al-Salamah MA, Al-Madani WH, Elbarbary MA. Systematic Review and Meta-analysis for the Use of Ultrasound versus Radiology in Diagnosing of Pneumonia. Crit Ultrasound J 2017;9:6.

45. Yousefifard M, Baikpour M, Ghelichkhani P, et al. Screening Performance Characteristic of Ultrasonography and Radiography in Detection of Pleural Effusion; a Meta-analysis. Emerg (Tehran) 2016;4:1-10.

46. Grimberg A, Shigueoka DC, Atallah ÁN, Ajzen S, Iared W. Diagnostic accuracy of sonography for pleural effusion: systematic review. Sao Paulo Med J 2010;128:90-95.

47. Fields JM, Davis J, Girson L, et al. Transthoracic Echocardiography for Diagnosing Pulmonary Embolism: a Systematic Review and Meta-analysis. J Am Soc Echocardiogr 2017;30:714-723.e4.

48. Bhatt M, Braun C, Patel P, et al. Diagnosis of deep vein thrombosis of the lower extremity: a systematic review and metaanalysis of test accuracy. Blood Adv 2020;4:1250-1264.

49. Da Costa Rodrigues J, Alzuphar S, Combescure C, Le Gal G, Perrier A. Diagnostic characteristics of lower limb 
venous compression ultrasonography in suspected pulmonary embolism: a meta-analysis. J Thromb Haemost 2016;14:1765-1772.

50. Huang H, Shen Q, Liu Y, Xu H, Fang Y. Value of variation index of inferior vena cava diameter in predicting fluid responsiveness in patients with circulatory shock receiving mechanical ventilation: a systematic review and meta-analysis. Crit Care 2018;22:204.

51. Si X, Xu H, Liu Z, et al. Does Respiratory Variation in Inferior Vena Cava Diameter Predict Fluid Responsiveness in Mechanically Ventilated Patients? A Systematic Review and Meta-analysis. Anesth Analg 2018;127:1157-1164.

52. Orso D, Paoli I, Piani T, Cilenti FL, Cristiani L, Guglielmo N. Accuracy of Ultrasonographic Measurements of Infe- rior Vena Cava to Determine Fluid Responsiveness: a Systematic Review and Meta-analysis. J Intensive Care Med 2020;35:354-363.

53. Long E, Oakley E, Duke T, Babl FE; Paediatric Research in Emergency Departments International Collaborative (PREDICT). Does Respiratory Variation in Inferior Vena Cava Diameter Predict Fluid Responsiveness: a Systematic Review and Meta-analysis. Shock 2017;47:550559.

54. Zhang Z, Xu X, Ye S, Xu L. Ultrasonographic measurement of the respiratory variation in the inferior vena cava diameter is predictive of fluid responsiveness in critically ill patients: systematic review and meta-analysis. Ultrasound Med Biol 2014;40:845-853. 